\title{
Un marco conceptual para la interpretación psicológica del juego infantil
}

\author{
ROSARIO ORTEGA
}

Universidad de Sevilla

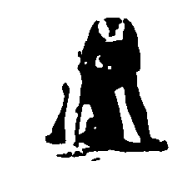

\section{Resumen}

El artículo plantea un análisis conceptual de la teoria del juego de Piaget y la compara críticamente con la teoria que se desprende del pensamiento vygotskiano sobre el tema. Aunque bay una elección por parte de la autora del trabajo de este último constructo teórico, el estudio está dirigido a resaltar aquellos aspectos del discurso piagetiano que considera fundamentales para elaborar una teoría psicológica del juego infantil, y a criticar aquellos otros que le parecen contradictorios o deficitarios.

Palabras clave: Asimilaciön deformante, Escenario lúdico, Conflicto cognitivo, Culturización.

\section{A conceptual frame for a psychological interpretation of child's play}

\section{Abstract}

This paper presents a conceptual analysis of Piaget's play theory and compares it with Vygotski's thought. Although a choice is made in favour of the latter theory, this study is aimed at bighlighting those aspects of Piaget's ideas which are basic to the development of a psychological theory of child's play, and to discuss those which we consider contradictory or incomplete.

Keywords: Strained assimilation, Play scene, Cognitive conflict, Culturization.

Dirección de la autora: Universidad de Sevilla.. Instituto de Ciencias de la Educación. Avda. Ramón y Cajal 1. 41005 Sevilla.

Original recibido: Octubre 1990. Revisión recibida: Marzo 1991. Aceptado: Mayo 1991. 


\section{INTRODUCCION}

El juego de los niños está comenzando a ser visto por educadores, padres y sociedad en general como una actividad natural cargada de posibilidades de aprendizaje y desarrollo.

La necesidad de modificar los esquemas tradicionales que relacionaban el aprendizaje con la transmisión de ideas o habilidades para su posterior dominio, y la tendencia a valorar las situaciones naturales y cotidianas como escenarios de desarrollo espontáneo, hacen pensar en el juego como un marco educativo privilegiado.

Se hace pues imprescindible la articulación de una teoría psicológica general sobre el juego que aporte al sistema educativo conceptos de referencia que justifiquen lo que intuitivamente comienza a ser aceptado como evidente, esto es, que el juego tiene amplias y precisas posibilidades de ser contexto natural para el aprendizaje, y por tanto, útil en la enseñanza.

Por nuestra parte, bajo una motivación fundamentalmente educativa, hemos investigado este tema y para ello hemos indagado en las teorías psicológicas que hoy se consideran básicas en la elaboración de modelos educativos, buscando un esquema conceptual de referencia suficientemente sólido que sirva de paradigma para la interpretación de los comportamientos lúdicos.

La reflexión que hoy presentamos sobre las perspectivas de Piaget y de Vygotski en el tema del juego pretende aportar datos sustantivos que nos lleven a una formulación teórica que caracterice psicológicamente el juego para, posteriormente, ver qué implicaciones educativas podemos inferir del mismo y qué uso podemos hacer de él en la práctica.

\section{UNA TEORIA ESTRUCTURAL}

La teoría de Piaget es un buen paradigma al que referirse para definir el juego y desde la cual indagar algunos puntos complejos e interesantes que la conducta lúdica sigue planteando, porque, como todo paradigma, es fructífero no sólo como plataforma básica de acuerdos, sino como referencia desde la cual modificar elementos en aras a la elaboración de constructos nuevos.

Hay tres trabajos fundamentales que tratan sobre el juego en la abundantísima obra de Piaget. El primero, de 1932, es un estudio sobre la moralidad de los niños y el desarrollo de sus ideas sobre la justicia y las convenciones sociales en el que Piaget trata del juego de reglas y define los juegos de los niños como «admirables instituciones sociales». En el segundo, Piaget (1946) aborda el tema del juego en un estudio sobre la formación del símbolo en el niño; en él se puede observar lo que Piaget llama una teoría estructural. Allí plantea la relación del juego con las estructuras cognitivas, proporcionándonos un estudio exhaustivo de la naturaleza del comportamiento lúdico y una clasificación general de los juegos infantiles. En estas dos obras encontramos ideas muy claras sobre qué es el juego para Piaget, y se nos informa de que éste no es otra cosa que una orientación personal en el funcionamiento de las estructuras cognitivas generales. Este 
matiz, esta orientación, es de naturaleza subjetiva y personal, y en términos de invariantes funcionales es básicamente asimilación deformante.

La evolución de los juegos infantiles se debe concebir como evolución de las estructuras básicas del conocimiento. De esta forma es lógico encontrar primero un juego de acción de características sensorio-motoras, posteriormente un juego simbólico y más tarde, los juegos reglados, en perfecta correspondencia con los grandes estadios del desarrollo cognitivo.

El tercer trabajo de Piaget sobre el tema, (Piaget, 1966), es un artículo en el que responde a la crítica que Sutton-Smith (1966) le plantea, en parte debido a la errónea interpretación que éste hace de ciertos postulados como el del egocentrismo, en parte a deficiencias teóricas en aspectos parciales que se pueden descubrir en ésta, si se interpreta la teoría piagetiana como explicativa de la totalidad de la conducta infantil.

De los tres, el trabajo más importante sobre el juego en general es el de 1946, en el que Piaget desarrolla su teoría de la función simbólica; a él nos vamos a referir en nuestra reflexión crítica tratando de comparar la perspectiva piagetiana con la de Vygotski.

\section{Criterios explicativos de las características psicológicas del juego}

Cuando Piaget intenta una explicación global sobre la naturaleza psicológica del juego infantil, to hace mediante dos vías: la primera a través del análisis de las características diferenciales del juego como comportamiento espontáneo en el niño, y la segunda a través del desarrollo evolutivo de las estructuras básicas del conocimiento y su paralelismo en el juego. De esta forma él pretende obtener una teoría general suficientemente explicativa del fenómeno. El análisis de las características diferenciales lo basará en los siguientes rasgos específicos:

\section{El subjetivismo}

Piaget tiende a establecer un camino evolutivo desde el autotelismo (máxima centración o finalidad en la acción propia), al egocentrismo (centración de carácter representativo, en el yo), y desde éste a la conducta social (descentración y capacidad de significación del pensamiento del otro). Esta visión de las cosas está siendo criticada en la actualidad (Flavell et all, 1968; Flavell, 1974; Flavell y Ross, 1984; Turiel, 1983; Enesco, 1985) y desde luego ya lo fue en los años de las publicaciones originales por autores como Vygotski $(1956,1986)$ o Wallon (Palacios, 1983), considerándose que no es sólo el nivel de centración en la acción o el pensamiento lo que define la madurez de la conducta, ni éste el factor más importante de las estructuras cognitivas.

$\mathrm{El}$ abandono del autotelismo como conducta primitiva, lo relaciona Piaget con la diferenciación en la acción del proceso asimilatorio y el acomodatorio, diferenciación que no sobrevendrá hasta pasado el primer año de vida, y que sucede de forma desigual en el proceso «serio" y en el lúdico. Efectivamente, en los llamados actos autotelicos del primer año (reacciones circulares primarias), hay poca diferenciación, al principio, entre lo asimilatorio y lo acomodatorio, pero a medida que ésta se va produciendo, la conducta «seria» deja de ser autotélica y pasa a ser heterotélica (centrada en las cosas), mientras que en el juego permanece un nivel de autotelismo 
superior y de predominio de lo asimilatorio frente a lo acomodatorio. Podríamos así decir que para Piaget el juego es siempre más subjetivo, más egocéntrico. Esto es especialmente así en la estructura del juego simbólico, que se convierte para Piaget en un reducto de fantasías personales cargadas de deseos insatisfechos y de loca imaginación. Sólo perderá su carácter de hipersubjetividad en el último escalón del proceso evolutivo de tal actividad, esto es, en el juego reglado, con la incorporación de la conciencia sobre la naturaleza de las normas y el sometimiento a las costumbres y convencionalidades del grupo de compañeros y de la tradición cultural.

\section{La espontaneidad}

La espontaneidad es estudiada por Piaget como segunda característica del juego, exponiendo que tanto la conducta indagatoria (experimentación y exploración intelectual), como la conducta científica, tienen características de conducta espontánea, frente a la conducta que deviene impuesta por la situación y la necesidad adaptativa obligada. Coloca así Piaget el juego y la actividad científica en una misma dimensión, si bien en polos de alguna manera opuestos. Veamos cómo lo plantea.

«Pero, ęlas investigaciones intelectuales primitivas del niño y las de la ciencia pura misma, no son también igualmente "espontáneas"? Si se quiere precisar $y$, por consecuencia, distinguir los «juegos superiores», que son la ciencia y el arte, de los juegos no "superiores», como los juegos a secas, no nos queda otra vez sino distinguir dos polos: un polo de actividad verdaderamente espontánea ya que no es controlada y un polo de actividad controlada por la sociedad o por la realidad». Piaget (1946), p. 201 de la edición de 1961.

El juego supone una espontaneidad no controlada, libre, mientras que el comportamiento científico es una espontaneidad controlada. De cualquier manera, el criterio espontaneidad es explicable si se interpreta el juego como la asimilación relajada del esfuerzo acomodatorio a la realidad o conducta obligada.

\section{El placer}

El tercer criterio o del "placer», es analizado por Piaget también en términos de contraposición a la conducta seria, la cual busca habitualmente una meta, un resultado útil. El placer es, dice Piaget, la cara afectiva del autotelismo, y supone la eliminación de la meta como motor de la acción.

El criterio del placer como rasgo definitorio del juego tampoco es un criterio diferencial específico, sino un nivel en un gradiente. Para Piaget, como para Vygotski, el juego es el lugar de satisfacción de deseos inmediatos. Pero mientras que Vygotski habla de deseos y necesidades epistemológicas y de acción, Piaget parece aceptar la interpretación psicoanalítica de que se trata de deseos de naturaleza yoica (compensaciones, desplazamientos, etc.). Como la espontaneidad, el placer representa la finalidad intrínseca del juego.

"El juego es una actividad "para el placer" mientras que la actividad seria tiene por meta un resultado útil, independientemente de su carácter agradable..." 
«El matiz más exacto es sin duda aquel que, en términos parecidos, Freud ha expresado por la oposición del "Lustprinzip" y del "Realitäsprinzip"

«En resumen, puede reducirse el juego a una búsqueda de placer, pero con la condición de concebir esta búsqueda como subordinada en sí misma a la asimilación de lo real al yo». Piaget (1946), p. 203 de la edición de 1961.

\section{El nivel de organización de la actividad lúdica}

El cuarto criterio es la falta de organización del juego, la carencia de estructura de esta actividad en oposición al comportamiento no lúdico. Aquí de nuevo Piaget parece renunciar al principio diseñado por él mismo y enuncia su idea de la falta de organización interna del pensamiento simbólico (precisamente por concebirlo al servicio de la vida afectiva y emocional), frente a la organización y desarrollo del conocimiento de la realidad o pensamiento lógico.

«Aquí también se puede comparar este criterio con una observación de Freud, según la cual el pensamiento simbólico (en el sentido del simbolismo inconsciente freudiano, del cual veremos precisamente el parentesco con el juego), no está "dirigido", por oposición con el pensamiento lógico, que obedece a una dirección sistemática». Piaget (1946), p. 203 de la edición de 1961.

Para Vygotski esto es muy distinto al considerar que todo juego dispone de reglas internas que regulan la acción bajo el control del significado.

Así lo podemos ver cuando dice:

«Es de señalar que el pequeño comienza con una situación imaginaria que inicialmente está muy cerca de la situación real. Reproduce exactamente esta última. Por ejemplo, una niña que está jugando con una muñeca repite casi de modo idéntico todo lo que su madre hace con ella. Esto significa que en la situación original las reglas operan de forma condensada y abreviada». Vygotski (1934/79), p. 156.

o cuando afirma:

«Pero lo que importa para el juego es la regla interna, propia, personal». Vigotsky (1933/80), p. 271.

\section{La resolución de conflictos}

Un quinto criterio sobre la naturaleza del juego es el ser «resolución ficticia de conflictos». Dice Piaget que el juego ignora los verdaderos conflictos y cuando los encuentra es para liberar al yo mediante una resolución de compensación o de liquidación, mientras la actividad seria se debate en conflictos de difícil solución, que obligan al sujeto a acomodar sus esquemas mentales y de acción a la realidad. Piaget, como Vygotski, piensa que el gran error de las teorías anteriores estuvo en no estudiar esta actividad en el contexto que le corresponde, esto es, inserta en el conjunto de conductas adaptativas y de desarrollo; en la estructura misma de determinación del cambio evolutivo. Pero de nuevo Piaget parece incluir los conflictos lúdicos en el área del mundo afectivo, mientras Vygotski acerca la problemática al mundo epistemológico o del conocimiento y considera que es 
la necesidad de saber lo que ocurre en su entorno y en los ámbitos no conocidos lo que empuja a jugar al niño explorando mundos nuevos; se trata de necesidades de conocer y hacer y no de caprichos emocionales.

Piaget va mostrando como cada una de las características que se habían descrito anteriormente como rasgos diferenciales del juego (Groos, Buytendijk, Claparède, Spencer, Hall), son perfectamente incluibles en lo que él denominaba la polarización de la asimilación en el proceso de funcionamiento de las invariantes funcionales (Flavell, 1979), esto es, una forma particularmente relajada de funcionamiento de la «maquinaria» básica del conocimiento. Veamos cómo lo expresa:

«Se puede, pues, afirmar que si la actividad y el pensamiento adaptados constituyen un equilibrio entre la asimilación y la acomodación, el juego empieza desde el momento en que la primera supera a la segundaw. Piaget (1946), p. 205 de la edición de 1961.

\section{ESTRUCTURA FRENTE A CONTENIDO}

Una de las críticas que hace Piaget a las teorías anteriores, es la miopía demostrada al no tratar de comprender la naturaleza de la estructura lúdica sino su contenido. El establece un diseño teórico que, efectivamente, parte del origen mismo de la organización psicológica, esto es, la acción exploradora e interactiva del sujeto con el mundo, que se va desarrollando hacia la complejización con la inclusión de elementos simbólicos, cada vez más abstractos y esquematizadores. El contenido concreto del pensamiento, tanto en el juego como en la conducta seria, será interpretado por Piaget como secundario. Lo que cuenta es el esqueleto lógico del enunciado, no el significado en sí. En el valor estructurante del contenido hay también diferencias entre las teorías que estamos analizando. Para Vygotski los contenidos que se representan en el juego simbólico tienen la función de ordenar, según las reglas del significado, la acción lúdica. Dichas reglas del significado simbólico están, como veremos, inscritas en la acción a través del sello cultural que la acción adquiere.

En lo que encontramos semejanza entre la teoría vygotskiana y piagetiana es en la consideración de la actividad como el origen, tanto del juego como de toda estructura básica de desarrollo; pero como trataremos de mostrar, ni los elementos que se toman en consideración son los mismos, ni el camino que éstos siguen es idéntico, y evidentemente el resultado es diferente.

Este concepto de juego, estrechamente vinculado a la acción espontánea, le permite a Piaget ajustar todos los rasgos atribuidos al mismo en una explicación unitaria: el placer por la acción, la repetición, la carencia de finalidad, el impulso subjetivo, la orientación hacia las cosas, etc., quedan satisfactoriamente aclaradas, a su modo de ver, como elementos diferenciales del juego, en la medida en que las características deformantes de la asimilación lúdica guían la incorporación de aspectos subjetivos al juego, y finalmente este subjetivismo es considerado por el autor de Ginebra el rasgo especial.

La segunda vía explicativa de la teoría piagetiana del juego infantil es la psicogenética o evolutiva, veamos cómo lo fundamenta: 


\section{EVOLUCION DE LOS JUEGOS Y DESARROLLO PSICOLOGICO}

A través de esta vía Piaget va a intentar articular una suerte de paralelismo entre la evolución de las estructuras básicas del conocimiento y las formas que adquiere el comportamiento lúdico infantil. Tratará Piaget de demostrar cómo llega el juego a ser de sensorio motor a simbólico, y de simbólico a reglado, y cómo en cada uno de estos estadios se mantiene la diferencia funcional entre el comportamiento serio y el lúdico, y el parecido de ambos como expresiones de estructuras epistemológicas.

Piaget considera que entre el juego de acción y el simbólico existe un innegable parentesco, una articulación lógica, diciendo que el juego simbólico es al juego de ejercicio lo que la inteligencia representativa es a la inteligencia sensorio motora (Piaget, 1946).

Entre la inteligencia sensorio-motora y el pensamiento representativo hay una relación de diacronía, y entre el juego sensorio motor y el simbólico también. A su vez entre inteligencia sensorio motora y juego de acción hay una relación sincrónica igual que entre juego simbólico e inteligencia representativa.

El juego simbólico surge, de igual forma que la inteligencia representativa, por la aparición dentro de la acción de la disociación entre el significante y el significado. Los indicios de las reacciones circulares terciarias se transforman en verdaderos significantes. Los signos verbales son los primeros significantes externos; la imagen mental, la primera separación entre la acción y el significado; la evolución de la imagen a través de la imitación (directa primero, diferida después), constituirán un signo.

El símbolo lúdico es también una imagen y por consiguiente es también imitación, esto es, el resultado de un proceso acomodatorio. Pero en el símbolo lúdico, la relación acomodación/asimilación se presenta de forma alterada, distinta a la fórmula de la imitación seria, por dos razones: en primer lugar, en la imitación seria hay correspondencia término a término objeto/significado, mientras que en el símbolo lúdico no; y en segundo lugar, la meta de la imitación seria es el ajustarse al objeto (meta acomodatoria), mientras que la meta del símbolo lúdico es el placer (meta asimilatoria de carácter subjetivo). En términos del propio Piaget, el juego crea una actitud "patética» y egocéntrica en lugar de objetiva. En definitiva, en el símbolo lúdico sólo hay asimilación del significado al yo.

«El juego simbólico no es otra cosa que el pensamiento egocéntrico en su estado puro. La condición necesaria para la objetividad del pensamiento es que la asimilación de lo real al sistema de las nociones adaptadas se encuentre en equilibrio permanente con la acomodación de estas mismas nociones a las cosas y al pensamiento de los otros sujetos». Piaget (1946), p. 227 de la edición de 1961.

Como veremos, ésta es una de las claves de la diferencia entre la teoría vygotskiana y la piagetiana del juego. Para ambos la naturaleza del símbolo es distinta. Para Vygotski (1933/1980) en el símbolo lúdico hay una estructura interna de adaptación al rol o a la situación representada que está en consonancia con la percepción, llena de sentido, del significado que para el niño tiene el objeto a representar. Esto impone unas normas al símbolo lú- 


\section{4}

dico que el niño tiene en cuenta y que permiten comprender la naturaleza del juego. De ahí que para Vygotski todo juego desde muy tempranamente es acción, símbolo y regla, en un conjunto coherente para el propio sujeto y que está cargado de sentido personal y cultural.

Para Piaget, en cambio, al relacionar juego y estructura cognitiva, despreciando el contenido que se representa, de tal forma interdependientes, cierra las puertas a análisis culturales y antropológicos que había iniciado él mismo y que están presentes en su trabajo de 1932 sobre la naturaleza social de los juegos, en el tema concreto de la adquisición de la conciencia moral (Piaget, 1932).

Esto lo vemos definitivamente cuando enuncia la diferencia estructural entre el juego y el pensamiento:

"Se sobreentiende que solamente la constitución de sistemas operacionales lógicos (reversibilidad de las transformaciones del pensamiento), morales (conservación de los valores) y espacio-temporales (organización reversible de las nociones físicas elementales), conducen a semejante equilibrio (equilibrio de las estructuras cognitivas), puesto que sólo la reversibilidad operativa permite al pensamiento conservar sus nociones a través de las fluctuaciones de lo real y pese al choque interesante de las novedades previstas" (Piaget, 1946, p. 227 de la edición de 1961).

Teniendo en cuenta la separación que Piaget hace del proceso de elaboración de esquemas mentales prelógicos del estadio preoperatorio y la definición que ha dado de la naturaleza simbólica del juego, nos parece importante afirmar que en alguna medida se equivocaba. Los símbolos lúdicos no son de naturaleza diferente al resto de las representaciones preoperativas, sino que también las representaciones lúdicas están sustentadas en las creencias y en las intuiciones (lo que se sabe, lo que se supone posible), y las ficciones (lo que se imagina) no son exactamente lo contrario de las creencias espontáneas, sino que están basadas así mismo en ellas, y mantienen con ellas una relación lógica, normativa, y psicológica de carácter experiencial.

Es nuestro trabajo (Ortega, 1988), hemos planteado una crítica a este supuesto piagetiano de que el símbolo lúdico es muy diferente de la creencia espontánea (saber adquirido y que se domina). Por el contrario encontramos que la ficción, la trama de los juegos, está en íntima relación con el «saber» y el «saber hacer" del que disponen los niños y, en este sentido, no se trata de dos procesos antagónicos, sino semejantes e interdependientes, y en gran medida complementarios. También Vygotski lo enuncia así:

«En el juego vemos el movimiento del niño en el plano puro de los significados bajo la libertad, la imaginación, la satisfacción de los deseos, etc. Eso es lo principal. Pero vemos eso mismo en la percepción real: la unión de lo visible con el significado-percepción del objeto" «... de manera que lo uno no se entiende sin lo otro" (Vygotski, 1933/80, p. 272).

Aunque aceptamos la teoría general del juego de Piaget como la única que analiza, basándose en la estructura interna de dicha actividad, la naturaleza del juego como un factor del desarrollo cognitivo, encontramos una cierta contradicción en lo que se refiere a la valoración de la representación lúdica como algo más relacionado con el mundo afectivo que con el mundo del pensamiento espontáneo o saber real sobre las cosas. 
Piaget abrió las posibilidades de análisis cognitivo del juego al relacionarlo con la organización y el desarrollo general del conocimiento; sin embargo, a partir de sus trabajos, paradójicamente el juego vuelve a ser encerrado en aspectos parciales y se confía poco en su valor como marco educativo. Creemos que se ha ido marginando, de alguna forma, la globalidad de la concepción de lo lúdico como una dimensión de desarrollo, esto es, como zona específica de desarrollo general del sujeto.

Creemos que el juego es un escenario donde se produce la acción y la representación del conflicto cognitivo, la perturbación responsable del desequilibrio, la permeabilidad frente a elementos nuevos que asimilar que alberga el proceso asimilatorio, y el ajuste acomodatorio de las estructuras antiguas y un cierto grado de equilibramiento fluido y flexible, dispuesto siempre a ser perturbado por otros elementos novedosos que en cualquier momento vengan a alterar el siempre dispuesto aparato cognitivo.

Visto así, entendemos que la relación juego y desarrollo cognitivo aporta mútiples posibilidades, tanto de aprendizaje espontáneo como de intervención educativa (Ortega, 1990). No basta, por tanto, ver el juego como un apéndice «divertido" de la dinámica del funcionamiento cognitivo, no basta tratar de encontrar en las organizaciones sociales de niños que juegan un reflejo de su propia concepción de la moral, de la justicia o de la convención social, creemos que es preciso establecer la importancia real del juego como escenario donde los niños reproducen y recrean los conocimientos y los esquemas de acción sobre el mundo que les rodea. Esto lo encontramos apuntado en la teoría de Vygotski y en los trabajos que bajo su guía científica realizaron Elkonin y sus colaboradores (Elkonin, 1980).

\section{UNA TEORIA SOCIO-COGNITIVA}

Para encontrar una explicación sobre el juego infantil en la escuela soviética, hay que remontarse a 1933, fecha en la que Vygotski supervisa las hipótesis de Elkonin y las defiende frente al academicismo psicológo; en aquella época escribió también sus «Apuntes para una conferencia de psicología de los párvulos" (Elkonin, 1980). Dicha conferencia fue pronunciada por Vygotski en 1933 en el Instituto Pedagógico de Leningrado, y publicada en "Voprosi Psikhologii» (Problemas de psicología) en 1966. En esos trabajos el juego se convierte en "clave» para el conocimiento de la estructura psicológica del niño preescolar.

«La relación del juego con el desarrollo es la de ser aprendizaje para el desarrollo. Tras el juego están los cambios de necesidades y los cambios de conciencia de carácter general. El juego es una fuente de desarrollo y crea zonas de evolución inmediata.» (Vygoski, 1933/1980, p. 269).

La articulación conceptual que realiza Vygotski de los tres elementos básicos del comportamiento: acción, símbolo y regla, nos permite inferir que él tenía una teoría del juego infantil suficientemente abarcativa como para explicar el papel de la actividad lúdica en la compleja organización psicológica del niño. En ella, la acción también es considerada origen, fundamento y concreción puntual del comportamiento; el simbolismo de carácter cultural y antropológico proporciona el significado a la acción; y la re- 
gla o normativa interna cohesiona en un todo lógico el resultado, permitiendo a los participantes de los juegos disponer de marcos estables en los cuales desplegar una acción que es clave para el desarrollo y el aprendizaje.

Un repaso a las ideas fundamentales de la teoría de Vygotski, nos va a permitir también señalar las características básicas y diferenciales del juego y su proceso evolutivo, pero esta vez tendremos que partir de los siguientes criterios:

\section{Bases filogenéticas y culturales del juego infantil}

Podemos decir que el enfoque soviético de la psicología del juego es un enfoque socio-histórico, no naturalista. El objetivo del juego es aprender del mundo de los adultos, de sus relaciones, sus actividades, transacciones y sistemas de organización y comunicación.

«En nuestra opinión el singular impacto que en el juego produce la actividad humana y las relaciones sociales evidencia que los temas de los juegos no se extraen únicamente de la vida de los mismos, sino que tienen un fondo social y no pueden ser un fenómeno biológico.» (Elkonin (1980), p. 74.)

Se presenta como una actividad que responde a las demandas de la sociedad en la que viven los niños; y de la que deben llegar a ser miembros activos. En sociedades primitivas, los niños no juegan a representaciones porque en éstas aún no están separados de la actividad laboral y adulta. Mead (1972) ha descrito que en sociedades como la melanesia, los niños hacen juegos representativos de aquellas actividades adultas que tienen un carácter oculto o más o menos prohibido (bodas, ritos, etc.). Así pues, un cierto origen cultural y antropológico hace que lo oculto, o lo que el niño sólo conoce a medias, sea motivo de interés lúdico. Es el deseo epistemológico de conocer lo que sólo se vislumbra, el que motiva la acción lúdica. Conocer cosas concretas que interesan porque nutren campos de interés, y no sólo porque relajan la dura estructura del pensamiento lógico. Veamos como lo dice Elkonin:

«El contenido del juego es el aspecto característico central, en él reconstruye el niño la actividad de los adultos y las relaciones que establece en su vida laboral y social.» Elkonin (1980), p. 32.

Estos argumentos son manejados por Elkonin como fundamentación de que el juego es un producto de la complejización de la estructura social y del papel del trabajo a lo largo de la historia de la humanidad, con la consecuencia de la separación de los niños del contexto social más específico de los adultos.

«En las distintas épocas de la historia, según fueran las condiciones sociohistóricas, geográficas y domésticas concretas de la vida, los niños practicaron juegos de diversa temática. Son distintos los temas de los juegos de los niños de las distintas clases, de los pueblos libres y de los pueblos oprimidos, de los pueblos nórdicos y de los meridionales, de los que habitan en terrenos bajos o en desiertos, de los hijos de obreros industriales, de los pescadores, de los ganaderos o de los agricultores.» (Elkonin, 1980, p. 32.)

En la tesis sociohistórica, Elkonin considera que la actividad laboral del hombre se ha diversificado y especializado de tal manera que la infancia ha 
quedado separada de dicha actividad. A su vez, el período de inmadurez y de preparación educativa para incorporarse al mundo laboral, se ha prolongado mucho y a esto hay que añadir la importancia del símbolo en la evolución de la cultura y la tecnología. Estos tres factores son los que han determinado que el juego y la actividad infantil se segreguen como formas simbólicas de carácter independiente, y que a través de ellas, se produzca el aprendizaje social que puede, de hecho, descubrirse en todo juego infantil.

La importancia del juego en la ontogénesis es correspondiente a la importancia del juego en la filogénesis; ambas son índices de la complejización, en el primer caso, de los sistemas laborales, económicos y sociales de la evolución del hombre, y en el segundo, de la complejidad de la estructuración del desarrollo individual, atendiendo a la inclusión de la cultura y lo social en la organización psicológica del individuo.

Recordemos (Rivière, 1984; Bruner, 1981) que para Vygotski, la educación y el desarrollo significan la culturización de los individuos y la incorporación de la historia de la humanidad, sus modelos y sus leyes, a la vida mental del sujeto. Así pues, en la psicología soviética del desarrollo, el juego tiene un origen filogenético que se relaciona con la evolución de los medios de producción y el desarrollo tecnológico de las sociedades, y un origen ontogenético que partiendo de la acción espontánea, desestructurada e inespecífica, incorpora el sentido social que el contexto da a la actividad específicamente humana a través del lenguaje y la adquisición de códigos comunicativos.

\section{Psicogénesis del comportamiento lúdico}

El origen del juego y de cualquier otra organización psicológica es la acción.

«Al igual que todas las funciones del conocimiento (el juego y la imaginación) surgen originariamente de la acción.» (Vygotski, 1934/1979, p. 143.)

Pero no se trata de la ciega acción del sujeto con el objeto, sino de un tipo particular de relación con los objetos, que los niños establecen bajo la orientación de los adultos. Piensa Elkonin que, aunque gran parte del uso de los objetos son de naturaleza utilitaria que describieron Kohler (1935) y Piaget (1946), otros usos no son sólo de carácter operativo, sino que son el resultado de aplicar un significado íntimo (personal) y socializador (cultural) a la actividad manipuladora.

"En el juego, las cosas pierden su fuerza determinante. El niño ve una cosa pero actúa prescindiendo de lo que ve. Así, alcanza una condición en la que el niño empieza a actuar independientemente de lo que ve." (Vygotski, $1934 / 1979$, p. 148.)

Esto explica que al uso esperado: «la cuchara es para comer», se les superponga un uso personal o lúdico: «la cuchara es un avión que va a aterrizar". Las acciones se convierten así en significantes de contenidos deseados o imaginados, de la simbolización o de los «como si», en los que el niño está inmerso. 
«En el juego el pensamiento está separado de los objetos y la acción surge a partir de las ideas más que de las cosas"... «La acción, de acuerdo con las reglas, está determinada por las ideas, no por los objetos en sí mismos." (Vygotski, 1934/1979, p. 149.)

Existe un proceso evolutivo interno en el uso de los objetos que va desde el uso literal de forma apropiada, a la sustitución por otro parecido para la misma acción, o la sobrevaloración del significado con independencia de los objetos que se usan para representar. Se establece una cadena de significantes desde el que más se aproxima al uso literal al que más se aleja; o dicho de otra forma, desde la mayor exclavitud a la mayor arbitrariedad en el uso de los objetos. Paralelamente a este proceso se da el de generalización de la relación significante/significado, desde la más rígida relación acción/objeto a una amplia relación acción/objeto con mayor arbitrariedad sobre qué objeto se utiliza.

¿Cuál es el papel del adulto en éste proceso? Dice Elkonin que es un papel de orientación, de irle dando al niño las pautas para que realice su propia trayectoria de complejización del símbolo y de la estructura de éste. El niño accede primero a la representación simbólica y posteriormente a la conciencia sobre la representación que está realizando. Para Elkonin es erróneo pensar en una relación entre el juego y el aprendizaje de las propiedades físicas de los objetos. Critica pues, el sentido de las investigaciones que desde Kohler (1925) hasta Bruner, Jolly y Sylva (1976), han relacionado el juego con la exploración en los aprendizajes instrumentales; por el contrario considera que el uso de objetos con carácter lúdico está siempre subordinado a la estructura simbólica de representación que se superpone a la acción con los objetos.

Los objetos del exterior tienen un papel en el juego desde el momento en que se insertan en la actividad orientadora y de búsqueda cognitiva que hay en éste. Durante los primeros tres años de vida se realiza una preparación de esta actividad orientadora que es la acción lúdica. Hay pues una trayectoria evolutiva de la acción lúdica, que va a desembocar en el florecimiento de lo que llamaremos juego sociodramático, o de protagonizar roles sociales. Dicha trayectoria podría enunciarse de la siguiente manera:

- Todas las premisas del juego se presentan en el desarrollo de la actividad del niño con objetos bajo los auspicios de los adultos y en actividad conjunta con ellos.

- Las primeras reproducciones simbólicas de acciones son con aquellos objetos que previamente han servido para construir acciones cargadas de sentido social, con la ayuda de un adulto.

- Apoyado por el adulto, el niño transfiere estas acciones a acciones con otros objetos, poniéndoles un nombre lúdico a los objetos después de haber operado con ellos y de que el adulto los haya considerado lúdicamente.

- Se adjudica a sí mismo nombre lúdico sólo después de que el adulto le haya atribuido a él un nombre lúdico, o a propuesta de él.

En definitiva, en la aparición de la estructura simbólica de los juegos, los adultos y la relación del niño con ellos tiene un valor determinante, convirtiendo la acción espontánea en acción o actividad plena de sentido so- 
cial, al incorporar el simbolismo cultural a la estructura compleja de la acción, en situaciones específicas conocidas como tales por los niños.

\section{Todo juego es de acción, simbólico y reglado}

Para la escuela soviética existe una fuerte relación entre toda actividad lúdica y el sistema de reglas que internamente la constituye. Entendemos que alude a la idea de que la voluntad (la trayectoria evolutiva de los deseos y las motivaciones), se forja en el camino de la aceptación/refutación de los sistemas normativos en los que el hombre se inserta y en la idea de que este camino no es independiente de la evolución cognitiva, moral o conductual del hombre. La regla es escuela de voluntades, dirá Vygotski, $(1933 / 1980)$ emulando a Spinoza.

En esta misma línea aporta la idea de la unidad en la relación ficciónregla, considerando que la ficción, sobre todo de estructura y contenido dramático, contiene una lógica del fenómeno humano, una lógica del suceso que es patrimonio natural del pensamiento del hombre desde siempre. Creemos que su dominio de la literatura en general y de la narrativa en particular, le lleva a considerar el código lingüístico-literario como un conjunto de abstracciones perfectamente enlazadas por un sistema interno de gran altura simbólica. Este código adquiere propiedades instrumentales en la constitución del pensamiento espontáneo.

El juego, siempre simbólico, siempre representativo, se ajusta a un código discursivo que se establece entre los jugadores y que no sólo da sentido a la acción, sino que permite el diálogo y la comprensión mutua, desde el momento en que hay un referente común, las reglas, a las cuales acudir para resolver cualquier conflicto entre los jugadores, y una acción común, considerada natural, dado el sentido de la acción. Esto es válido tanto para los llamados por Piaget, juegos de reglas (las canicas por ejemplo), como para los juegos simbólicos; jugar a "casitas» puede ser muy diferente para cada niña, a la vez que hay elementos universales, tales como la reproducción de roles sociales familiares.

«La trayectoria central del desarrollo del juego va de la situación ficticia patente con regla y objetivo lábiles, a la situación ficticia con reglas y objetivo patentes y ficción, reglas y objetivo constantes (el juego a las muñecas y el juego al ajedrez son polos del desarrollo.» (Vygotski, 1933/1980, p. 273.)

Se puede establecer un gradiente de subjetividad-objetividad y un gradiente de flexibilidad-rigidez de las reglas del juego, en proporción universal al «quantum» de simbolismo/regla, pero es difícil encontrar juegos que no estén compuestos de acción, símbolo y regla a la vez.

\section{SINTESIS Y CONCLUSIONES}

La teoría de Piaget establece los principios psicológicos básicos para formular un concepto del juego infantil que define éste como parte del proceso cognitivo en particular y de desarrollo en general; sin embargo la consideración del juego como asimilación deformante y por tanto como subjetivo y egocéntrico, dificultan de hecho su consideración de marco social 
para la adquisición de conocimientos. Por el contrario, considerando el juego infantil como un comportamiento, que tiene naturaleza simbólica, y que es, por tanto, una conducta representativa que se organiza en torno a unas reglas, es mas fácil elaborar una teoría psicológica sobre la naturaleza del juego que posibilite una explicación del desarrollo de este comportamiento acorde con la teoría general del desarrollo y el aprendizaje infantil, y que permita su utilización como marco educativo.

Encontramos en la perspectiva vygotskiana un complemento de la teoría estructural de Piaget que, al coste de refutar algunos presupuestos piagetianos, como el paralelismo entre desarrollo cognitivo y esquemas lógicos y el abuso del concepto de egocentrismo intelectual, permite comprender mejor el desarrollo de esta interesante actividad infantil.

En definitiva, podemos rescatar de la teoría piagetiana y confirmar desde la teoría vygotskiana, los siguientes elementos:

La acción como estructura psicogenética básica del despliegue de todo comportamiento complejo, pero no una acción ciega ni orientada por el propio objeto, sino una acción iluminada por la interacción del niño con los adultos u otros compañeros, y culturalmente marcada por el uso y la intención que históricamente el hombre da al uso de instrumentos, y la mediación del lenguaje. Todo juego es pues comunicación.

El subjetivismo de la acción lúdica, que está cargada de símbolos personales, pero también, sometida a las convenciones y normas generales y puntuales que todo juego incluye y que permite la comunicación y el diálogo dentro del marco de la ficción estando, por tanto, relativamente exento de egocentrismo intelectual. Pensamos que el juego es más una escuela de auto control del pensamiento que de exuberante centración propia.

La evolución de los juegos en paralelo con las formas básicas que el niño tiene de organizar sus conocimientos del mundo y de cómo actuar dentro de él, y en este sentido confirmar los tres grandes períodos del desarrollo lúdico, incluyendo entre el primer juego simbólico (2-4 años) y el juego reglado (8-10 años), un estadio específico: el de los juegos sociodramáticos o juegos de representación de papeles sociales.

La articulación crítica de estos dos constructos teóricos, nos ha proporcionado un marco que nos ha permitido explorar de una forma concreta el juego infantil de contenido simbólico-social (Ortega, 1988), en el que hemos encontrado cómo los niños despliegan sus conocimientos y los estructuran produciendo aprendizajes nuevos, y modificando sus estructuras cognitivas.

Sin embargo, creemos que se hace necesario el trabajo de análisis y conceptualización en un marco teórico para delimitar la naturaleza específica del juego infantil, y esto no sólo con los estudios experimentales, sino incorporando la línea socio-histórica que la escuela vygotskiana propone.

\section{Referencias}

Bretherton, I. (1984). Symbolic Play. Academic Pres. Orlando. Florida.

BRUNER, J. (1981). Vygotski: una perspectiva histórica y conceptual. En Infancia y Aprendizaje, 14, 3-17.

BRUNER, J. (1984). Juego, pensamiento y lenguaje. En Linaza, J. (1984): (Comp): Jerome Bruner, Acción, pensamiento y lenguaje. Ed. Alianza. Madrid. 
Bruner, J.; Jolly, A. y Silva, K. (1976). Play: its role in development and evolution. Penguin Nueva York.

BRUNER, J. y KOSLKOWSKY, B. (1972). Visually preadapted constituents of manipulatory action. Perception, 1, 3-14.

ELKonIN, D. B. (1980). Psicología del juego. Ed. Pablo del Río. Madrid.

ENESCO, I. (1985). Una revisión del concepto de egocentrismo espacial en tareas de adopción de perspectivas. Infancia y Aprendizaje, 30, 81-89.

Flavell, J. H. (1974). The development of inference about others. En T. Mishel (Ed.): Understanding others persons. Blackwell. Oxford.

Flavell, J. H. (1979). La psicologia evolutiva de J. Piaget. Paidos, Buenos Aires.

Flavell, J. H.; BotKIN, P. A.; FRY, C. L.; WRIGHT, J. W.; JARVIS, P. E. (1986). The development of the role-taking and communication skills in children. Wiley. Nueva York.

Flavell, J. H. y Ross, L. (1981). Social cognitive development. Cambridge University Press. Cambridge.

ORTEGA, R. (1988). Juego y conocimientos sociales. Tesis Doctoral. Universidad de Sevilla.

ORTEGA, R. (1990). Jugar y aprender. Ed. Diada. Sevilla.

Palacios, J. (1983). Henri Wallon. En J. Palacios, M. Carretero y A. Marchesi: Psicología evolutiva. Alianza. Madrid.

PiAgeT, J. (1932). En castellano: El criterio moral en el niño. Ed. Fontanella: Barcelona, 1971.

PIAGET, J. (1946). En castellano: La formación del símbolo. Ed. F.C.E. México, 1961.

PiageT, J. (1966). Response to Brian Sutton-Smith. Psychological Review, 73 111-112.

RIVIERE, A. (1984). La psicología de Vygotski: sobre la larga proyección de una corta biografía. Infancia y Aprendizaje. 27-28, 7-86.

TURIEL, E. (1983). The development of social knowledge. Cambridge. University Press. En castellano. Ed. Debate. Madrid, 1984.

VYGOTSKI, L. S. (1934). El desarrollo de los procesos psicológicos superiores. En castellano. Ed. Crítica. Barcelona, 1979.

VYGOTSKI, L. S. (1934). La imaginación y el arte en la infancia. En castellano. Ed. Akal, 1982, Madrid.

VYGOTSKI, L. S. (1956). Aprendizaje y desarrollo intelectual en la edad escolar. En Vygotski, Luria y Leontiev: Psicologia y Pedagogía. En castellano (1986). Madrid. Ed. Akal.

VYGOTSKI, L. S. (1933/1980). Apuntes para una conferencia sobre la psicología de los preescolares. En Elkonin 1980 Ed. Pablo del Río.

Wallon, H. (1941). L'evolution psychologique de l'enfant. En castellano. Ed. Grijalbo. Madrid, 1976.

\section{Extended summary}

Piaget's theory on the origin and development of children's play set up a pattern on the nature of such complex and interesting behaviour. This pattern links, epistemologically, play to cognitive structures and to its evolution throughout the different play stages. Piaget's analysis is based on the definition of play behaviour in terms of strainning assimilation, as opposed to a «serious» behaviour, controlled by a continuing process of accommodation which redresses the structures of knowledge. However, we do not altogether agree with this assumption.

Piaget's theory shows a tendency towards subjectivism and individualism due to: the influence of the idea that preschool children are self-centred and little prepared for social interaction, and to the need to oppose play to a serious organization of cognitive structures.

On the other hand, Piaget tends to analyze structures separately from their content. This in our view contradicts his own attempts to study play in connection with the development of knowledge.

Piaget thinks that symbolic play performs a major function as a «compensation of the conflicts of the self». Therefore, from a psychoanalytic view his analysis of symbolic play is directed to the field of individual fantasy and the unreality of thought. 
In this paper, a criticism of Piaget's theory is presented based on Vygotski's sociocultural theory on child's play. Vygotski's theory explains that these activities take place in "areas of close development" in which specific learning favours cognitive development. In this way, it provides important information for the understanding of play as a cognitive and spontaneous learning process.

The role ascribed to children's play by the Soviet school is also analyzed. That is, as a framework to situate the influence exerted by society on the orientation of "meaning" in the construction of children's thoughts.

Finally, an analysis of cognitive development is proposed which takes into account the role of the content and considers learning and development as a unit. This would allow to formulate a theory on the role of play in education, both in the school and at home, which would be the purpose of this theoretical study. 